International Journal of Pure and Applied Mathematics

Volume 95 No. 3 2014, 387-399

ISSN: 1311-8080 (printed version); ISSN: 1314-3395 (on-line version)

url: http://www.ijpam.eu

doi: http://dx.doi.org/10.12732/ijpam.v95i3.6

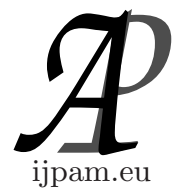

\title{
PROJECT SCHEDULING PROBLEMS UNDER FUZZY ENVIRONMENT: A NEW SOLUTION APPROACH
}

\author{
M. Shanmugasundari ${ }^{1 \S}, \mathrm{K}$. Ganesan ${ }^{2}$ \\ ${ }^{1,2}$ Department of Mathematics \\ Faculty of Science Humanities \\ SRM University \\ Kattankulathur, Chennai, 603203, INDIA
}

\begin{abstract}
In this paper we propose a new method for the solution of project scheduling problems under fuzzy environment. The duration of all fuzzy activities are represented as trapezoidal fuzzy numbers. We compute several project characters such as earliest times, latest times, slack times and various floats for each fuzzy activity in the project scheduling problems. Numerical examples are provided to illustrate the efficiency of the proposed method.
\end{abstract}

AMS Subject Classification: 15A09, 65F05, 65G30

Key Words: fuzzy number, trapezoidal fuzzy number, fuzzy arithmetic, fuzzy ranking, project network, critical path, total float, earliest start, latest finish

\section{Introduction}

Scheduling of activities and the control of their flows through a production process play most significant role in any modern manufacturing systems. Project scheduling is a complex decision making problem because of conflicting goals, limited resources and the difficulty in accurately modeling real world problems. Critical Path Method (CPM) and Project Evaluation and Review Technique

Received: April 23, 2014

(C) 2014 Academic Publications, Ltd.

$\S_{\text {Correspondence author }}$ url: www.acadpubl.eu 
(PERT) are widely recognized valuable tools for the planning and scheduling of large projects. In CPM a deterministic data for activity time is used where as in PERT random time data's are employed. However in reality, due to the uncertainty of information as well the variation of management scenario, it is often difficult to obtain the exact activity time estimates of all activities. Thus, the conventional approaches, both deterministic and random process, tend to be less effective in conveying the imprecision or vagueness nature of the linguistic values. One alternative to handle the uncertainty associated with the processing time is to use fuzzy techniques.

Several authors such as Chanas et.al [2, 3], Chang et.al[4], Chen et.al $[6,7]$,Kuchta [13], Lin et.al[15, 16], Macahon [19], and Shahsavari et.al [24, 25] etc proposed different methods for the solution of fuzzy critical path problems.The first method called Fuzzy Project Evaluation and Review Technique was proposed by Chanas and Kamburowski [2]which deals with the project completion time in the form of a Fuzzy set in the time space. Chanas and Zielinski [3] proposed a method to make critical path analysis in the network with fuzzy activity times (interval activity times, fuzzy numbers of L-R type). Dubois et al [8] assigns a different level of importance to each activity on a critical path for a randomly chosen set of activities. Gazdiak [9] developed a Fuzzy network of a priori unknown project to estimate the activity duration and used Fuzzy algebraic operators to calculate the duration of the project and its critical path. Nasution [21] proposed a fuzzy critical path method by considering the interactive fuzzy subtraction and by observing that only the nonnegative part of the fuzzy numbers can have physical interpretation. Shakeela and Ganesan [26] proposed a new algorithm to tackle fuzzy project network problems with out defuzzifying the fuzzy activity durations. Slyeptsov and Tyshchuk [29] presented an efficient method of computation of fuzzy time windows for late start and finish times of operations in the problem of fuzzy network.

By using a new ranking method and a new arithmetic operations for trapezoidal Fuzzy numbers, we propose a new method to solve fuzzy project scheduling problems without converting in to classical project networking problems. The rest of this paper is organized as follows:

In Section 2, we recall the basic concepts and the results of trapezoidal fuzzy numbers and their arithmetic operations. In Section 3, we introduce the fuzzy project network problems with trapezoidal fuzzy numbers and related results. We propose a new algorithm to find various fuzzy floats and fuzzy critical path and hence the fuzzy optimum duration of the project network. In Section 4, numerical examples are provided to illustrate the theory developed in this paper. 


\section{Preliminaries}

The aim of this section is to present some notations, notions and results which are of useful in our further considerations.

\subsection{Fuzzy Numbers}

Definition 2.1. A fuzzy set $\tilde{A}$ defined on the set of real numbers $\mathbf{R}$ is said to be a fuzzy number if its membership function $\tilde{A}: \mathbf{R} \rightarrow[0,1]$ has the following characteristics:

(i). $\tilde{A}$ is convex, that is $\tilde{A}\left(\lambda x_{1}+(1-\lambda) x_{2}\right) \geq \min \left\{\tilde{A}\left(x_{1}\right), \tilde{A}\left(x_{2}\right)\right\}$, for all $x_{1}, x_{2} \in \mathbf{R}$ and $\lambda \in[0,1]$

(ii). $\tilde{A}$ is normal i.e. there exists an $x \in \mathbf{R}$ such that $\tilde{A}(x)=1$.

(iii). $\tilde{A}$ is piecewise continuous.

Definition 2.2. A fuzzy number $\tilde{A}$ on $\mathbf{R}$ is said to be a trapezoidal fuzzy number if its membership function $\tilde{A}: \mathbf{R} \rightarrow[0,1]$ has the following characteristics:

$$
\tilde{A}(x)=\left\{\begin{array}{l}
\frac{x-a_{1}}{a_{2}-a_{1}}, \quad \text { for } \quad a_{1} \leq x \leq a_{2} \\
1, \text { for } \quad a_{2} \leq x \leq a_{3} \\
\frac{a_{4}-x}{a_{4}-a_{3}}, \quad \text { for } \quad a_{3} \leq x \leq a_{4} \\
0, \quad \text { otherwise }
\end{array}\right.
$$

We denote this trapezoidal fuzzy number by $\tilde{A}=\left(a_{1}, a_{2}, a_{3}, a_{4}\right)$. We use $F(\mathbf{R})$ to denote the set of all trapezoidal fuzzy numbers. Also if $\left[a_{2}, a_{3}\right]$ represents the core, $\alpha=\left(a_{2}-a_{1}\right)$ represents the left spread and $\beta=\left(a_{4}-a_{3}\right)$ represents the right spread of the trapezoidal fuzzy number $\tilde{A}$, then the trapezoidal fuzzy number $\tilde{A}$ can be represented by the triplet $\tilde{A}=\left(a_{1}, a_{2}, a_{3}, a_{4}\right)=$ $\left(\left[a_{2}, a_{3}\right], \alpha, \beta\right)$.

\subsection{Ranking of Trapezoidal Numbers}

An efficient approach for comparing the fuzzy numbers is by the use of a ranking function $\mathrm{R}: F(R) \rightarrow R$ which maps each trapezoidal fuzzy number into a real number, where a natural order exists 
For every trapezoidal fuzzy number $\tilde{A}=\left(a_{1}, a_{2}, a_{3}, a_{4}\right)=\left(\left[a_{2}, a_{3}\right], \alpha, \beta\right) \in$ $F(\mathbf{R})$, ranking function $\mathrm{R}: F(R) \rightarrow R$ is defined by graded mean as

$$
\mathrm{R}(\tilde{A})=\left(\frac{a_{2}+a_{3}}{2}\right)+\left(\frac{\beta-\alpha}{4}\right) .
$$

Now for any two trapezoidal fuzzy numbers $\tilde{A}=\left(a_{1}, a_{2}, a_{3}, a_{4}\right)=\left(\left[a_{2}, a_{3}\right]\right.$, $\left.\alpha_{1}, \beta_{1}\right), \tilde{B}=\left(b_{1}, b_{2}, b_{3}, b_{4}\right)=\left(\left[b_{2}, b_{3}\right], \alpha_{2}, \beta_{2}\right) \in F(\mathbf{R})$, we have the following comparison

(i). $\tilde{A} \succeq \tilde{b}$ if and only if $\mathbf{R}(\tilde{A}) \geq \mathbf{R}(\tilde{B})$

(ii). $\tilde{A} \preceq \tilde{B}$ if and only if $\mathbf{R}(\tilde{A}) \leq \mathbf{R}(\tilde{B})$

(iii). $\tilde{A} \approx \tilde{B}$ if and only if $\mathrm{R}(\tilde{A})=\mathbf{R}(\tilde{B})$

(iv). $\tilde{A}-\tilde{B} \approx \tilde{0}$ if and only if $\mathbf{R}(\tilde{A})-\mathbf{R}(\tilde{B})=0$

A trapezoidal Fuzzy number $\tilde{A}=\left(a_{1}, a_{2}, a_{3}, a_{4}\right)=\left(\left[a_{2}, a_{3}\right], \alpha, \beta\right) \in F(\mathbf{R})$ is said to be positive if $\mathbf{R}(\tilde{A})>0$ and is denoted by $\tilde{A} \succ \tilde{0}$. Also $\tilde{A} \prec \tilde{0}$ if $\mathbf{R}(\tilde{A})<0$ and $\tilde{A} \approx \tilde{0}$ if $\mathbf{R}(\tilde{A})=0$. If $\mathbf{R}(\tilde{A})=\mathbf{R}(\tilde{B})$ then the trapezoidal fuzzy numbers are said to be equivalent and is denoted by $\tilde{A} \approx \tilde{B}$.

\subsection{Arithmetic Operations on Trapezoidal Fuzzy Numbers}

Ming Ma et al. [20] have proposed a new fuzzy arithmetic based upon both location index and fuzziness index functions. The location index number is taken in the ordinary arithmetic, whereas the fuzziness index functions are considered to follow the lattice rule which is least upper bound in the lattice $L$. That is for $a, b \in L$ we define $a \vee b=\max \{a, b\}$ and $a \wedge b=\min \{a, b\}$.

Now for any two arbitrary trapezoidal fuzzy numbers $\tilde{A}=\left(a_{1}, a_{2}, a_{3}, a_{4}\right)=$ $\left(\left[a_{2}, a_{3}\right], \alpha_{1}, \beta_{1}\right)$,

$\tilde{B}=\left(b_{1}, b_{2}, b_{3}, b_{4}\right)=\left(\left[b_{2}, b_{3}\right], \alpha_{2}, \beta_{2}\right) \in F(\mathbf{R})$ and for $*=\{+,-, \times, \div\}$, the arithmetic operations on $\tilde{A}$ and $\tilde{B}$ are defined as

$$
\begin{aligned}
\tilde{A} * \tilde{B} & =\left(a_{1}, a_{2}, a_{3}, a_{4}\right) *\left(b_{1}, b_{2}, b_{3}, b_{4}\right)=\left(\left[a_{2}, a_{3}\right], \alpha_{1}, \beta_{1}\right) *\left(\left[b_{2}, b_{3}\right], \alpha_{2}, \beta_{2}\right) \\
& =\left(\left[a_{2}, a_{3}\right] *\left[b_{2}, b_{3}\right], \max \left\{\alpha_{1}, \alpha_{2}\right\}, \max \left\{\beta_{1}, \beta_{2}\right\}\right) .
\end{aligned}
$$

In particular for any two $\tilde{A}$ and $\tilde{B} \in F(\mathbf{R})$, we define

(i). Addition:

$$
\begin{aligned}
\tilde{A}+\tilde{B} & =\left(\left[a_{2}, a_{3}\right]+\left[b_{2}, b_{3}\right], \max \left\{\alpha_{1}, \alpha_{2}\right\}, \max \left\{\beta_{1}, \beta_{2}\right\}\right) \\
& =\left(\left[a_{2}+b_{2}, a_{3}+b_{3}\right], \max \left\{\alpha_{1}, \alpha_{2}\right\}, \max \left\{\beta_{1}, \beta_{2}\right\}\right) .
\end{aligned}
$$


(ii). Subtraction:

$$
\begin{aligned}
\tilde{A}-\tilde{B} & =\left(\left[a_{2}, a_{3}\right]-\left[b_{2}, b_{3}\right], \max \left\{\alpha_{1}, \alpha_{2}\right\}, \max \left\{\beta_{1}, \beta_{2}\right\}\right) \\
& =\left(\left[a_{2}-b_{3}, a_{3}-b_{2}\right], \max \left\{\alpha_{1}, \alpha_{2}\right\}, \max \left\{\beta_{1}, \beta_{2}\right\}\right) .
\end{aligned}
$$

(iii). Scalar Multiplication:

$$
\begin{aligned}
k \tilde{A} & =k\left(\left[a_{2}, a_{3}\right], \alpha_{1}, \beta_{1}\right) \\
& =\left\{\begin{array}{l}
\left(\left[k a_{2}, k a_{3}\right], \alpha_{1}, \beta_{1}\right), k \geq 0 \\
\left(\left[-k a_{3},-k a_{2}\right], \alpha_{1}, \beta_{1}\right), k<0
\end{array}\right.
\end{aligned}
$$

\section{Main Results}

Fuzzy Critical Path Method (FCPM) provides a graphical view of the project, to predict the time required to complete the project, to identify the critical activities in the project.

\subsection{Notations and Meaning}

$F \tilde{E} S_{1}=(0,0,0,0):$ The earliest fuzzy starting time of the initial event $\tilde{v}_{1}$

$F \tilde{E} S_{j} \quad$ : The earliest start Fuzzy time of event $\tilde{v}_{j}$

$$
=\max \left\{F \tilde{E} S_{i}+F \tilde{E} T_{i j} / i \in N P(j), j \neq 1, j \in N\right\}
$$

$F \tilde{L} F_{i}=$ The latest finish Fuzzy time of event $\tilde{v}_{i}$

$$
=\min \left\{F \tilde{L} F_{k}-F \tilde{E} T_{j k} / k \in N S(j), j \neq n, j \in N\right\}
$$

$F \tilde{T}_{i j}=$ The total float of Fuzzy activity $\tilde{a}_{i j}$

$$
=F \tilde{L} F_{j}-\left(F \tilde{E} S_{i}+F \tilde{E} T_{i j}\right), 1 \leq i \leq j \leq n ; i, j \in N
$$

$$
\begin{aligned}
\operatorname{FCPM}\left(p_{k}\right)= & \text { The Fuzzy completion time of the path } p_{k} \\
& \text { in a Fuzzy project network. } \\
& \sum_{1 \leq i \leq j \leq n} F \tilde{T}_{i j}, p_{k} \in P ; i, j \in p_{k}
\end{aligned}
$$


Theorem 3.1. Assume that the Fuzzy activity times of all activities in a project network are trapezoidal Fuzzy number, then there exists fuzzy Critical Path in the project network.

Proof. Let $F \tilde{E} S_{1}=(0,0,0,0)$ be the starting time of the project network. Then $F \tilde{E} S_{j}$ of the node can be calculated by the sum of $F \tilde{E} S_{i}$ and $F \tilde{E} T_{i j}$, where, $i \in N P(j), j=2,3, \ldots \ldots . n$. Then there must uniquely exist a maximal $F \tilde{E} S_{j}$ such that $F \tilde{E} S_{j}=\max \left\{F \tilde{E S}_{i}+F E \tilde{T}_{i j}, i \in N P(j), j \neq 1, j \in N\right\}$. Further more let $F \tilde{L} F_{n}=F \tilde{E} S_{n}$ to indicate the latest and earliest start of the last node of the project are same. Then $F \tilde{L} F_{j}$ of the jth node can be calculated by subtracting $F \tilde{E} T_{j k}$ from $F \tilde{L} F_{k}$, where $k \in N S(j)$ and $j=n-1, n-2, \ldots, 3,2,1$. Similarly there uniquely exists a minimal $F \tilde{L} F_{j}$ such that,

$$
F \tilde{L} F_{j}=\min \left\{F \tilde{L} F_{k}-F \tilde{E} T_{j k}, k \in N S(j), j \neq n, j \in N\right\}
$$

and so is $F \tilde{T}_{i j}$ also true for any $p_{k} \in P$ such that $F C P M\left(p_{k}\right)$ is unique and atleast there exists a path $p_{c}$ such that $F C P M\left(p_{c}\right)=\max \left\{F C P M\left(p_{i}\right) / p_{i} \in P\right\}$. Hence the proof.

\subsection{Proposed Algorithm}

Let $F \tilde{E} S_{i}$ and $F \tilde{L} S_{i}$ be the earliest Fuzzy event time and the latest Fuzzy event time for event i respectively.Functions that define the earliest starting times, latest starting times and floats in terms of Fuzzy activity durations are convex, normal whose membership functions are piecewise continuous, hence the quantities such as earliest Fuzzy event time $F \tilde{E} S_{i}$ the latest Fuzzy event time $F \tilde{L} S_{i}$ and floats $F \tilde{T}_{i j}$ are also trapezoidal Fuzzy numbers for an event i respectively.

Step 1. Identify each activity along with fuzzy duration in a Fuzzy project.

Step 2. Applying a Fuzzy ranking function, determine the precedence relationships of all fuzzy activities.

Step 3. Construct the fuzzy project network.

Step 4. Let $F \tilde{E} S_{1}$ be the earliest Fuzzy event time and $F \tilde{L} S_{1}$ be the latest Fuzzy event time for the initial event $\tilde{v}_{1}$ of the project network and assume that $F \tilde{E} S_{1}=\tilde{0}$. Compute the earliest Fuzzy event time $F \tilde{E} S_{j}$ of the event $\tilde{v}_{j}$ by using formula,

$$
F \tilde{E} S_{j}=\max \left\{F \tilde{E} S_{i}+F \tilde{E} T_{i j}\right\}, i \in N
$$


Step 5. Let be the earliest Fuzzy event time and be the latest Fuzzy event time for the terminal event of the Fuzzy project network and assume that . Compute the latest Fuzzy event time of the event using the formula,

$$
F \tilde{L} S_{i}=\min \left\{F \tilde{L} S_{j}-F \tilde{E} T_{i j}\right\}, j \in N
$$

Step 6. Compute the total float of each Fuzzy activity using the following formula,

$$
F \tilde{T}_{i j}=\left\{F \tilde{L} S_{j}-F \tilde{E} S_{i}-F \tilde{E} T_{i j}\right\}
$$

Step 7. If then activity is said to be a fuzzy critical activity. A path of longest fuzzy duration consists of critical activities is called a fuzzy critical path.

Step 8. The length of the fuzzy critical path is the maximum time required to complete the fuzzy project and is the optimal project duration of the fuzzy project network.

\section{Numerical Examples}

Example 4.1. Let us consider an example discussed by Ravi Shankar et.al [22]. Suppose that there is a project network, as Figure 2, with the set of events $\tilde{V}=\{1,2,3,4,5\}$, the fuzzy activity time for each activity is shown in Table 1.All the durations are in hours expressed as trapezoidal Fuzzy numbers.

Table 1: Duration of each fuzzy activity in a fuzzy network problem

\begin{tabular}{|c|c|c|c|c|}
\hline Activity & $1-2$ & $1-3$ & $2-3$ & $1-4$ \\
\hline Duration & $(10,15,15,20)$ & $(30,40,40,50)$ & $(30,40,40,50)$ & $(15,20,25,30)$ \\
\hline Activity & $2-5$ & $3-5$ & $4-5$ & \\
\hline Duration & $(60,100,150,180)$ & $(60,100,150,180)$ & $(60,100,150,180)$ & \\
\hline
\end{tabular}

For all activities in this fuzzy project, convert the given fuzzy activity time $\tilde{A}=\left(a_{1}, a_{2}, a_{3}, a_{4}\right)$ in the form of $\tilde{A}=\left(\left[a_{2}, a_{3}\right], \alpha_{1}, \beta_{1}\right)$ as follows

Fuzzy critical path is $1 \rightarrow 2 \rightarrow 3 \rightarrow 5$. The fuzzy project duration is the length of the fuzzy critical path. Hence the optimum fuzzy project duration is $(115,155,205,235)$ fuzzy hours, where as Ravi Shankar et.al [22] have obtained the same critical path without specifying the project duration. 
Table 2: Duration of each fuzzy activity in a fuzzy network problem

\begin{tabular}{|c|c|c|c|c|}
\hline Activity & $1-2$ & $1-3$ & $2-3$ & $1-4$ \\
\hline Duration & $([15,15], 5,5)$ & $([40,40], 10,10)$ & $([40,40], 10,10)$ & $([20,25], 5,5)$ \\
\hline Activity & $2-5$ & $3-5$ & $4-5$ & \\
\hline Duration & $([100,150], 40,30)$ & $([100,150], 40,30)$ & $([100,150], 40,30)$ & \\
\hline
\end{tabular}

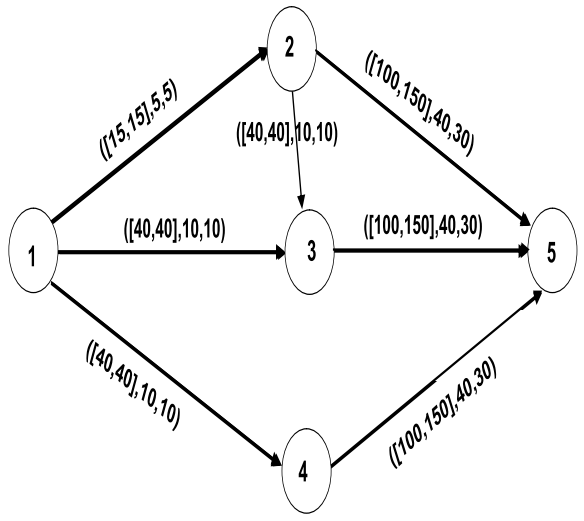

Figure 1:Fuzzy project network

Example 4.2. Now let us consider another example discussed by Usha Madhuri et.al [30]. Suppose that there is a project network, as Figure 2, with the set of events $\tilde{V}=\{1,2,3,4,5,6\}$ The fuzzy activity time for each activity time for each activity as shown in Table 4 .All the durations are in hours expressed as trapezoidal Fuzzy numbers.

For all activities in this fuzzy project, convert the given fuzzy activity time $\tilde{A}=\left(a_{1}, a_{2}, a_{3}, a_{4}\right)$ in the form of $\tilde{A}=\left(\left[a_{2}, a_{3}\right], \alpha_{1}, \beta_{1}\right)$ as follows

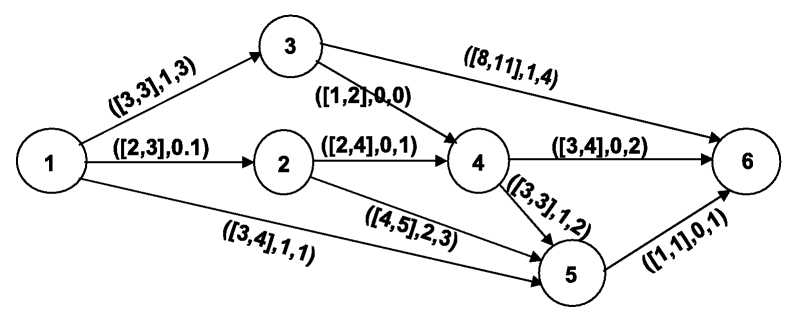

Figure 2 :Fuzzy project network

Fuzzy critical path is $1 \rightarrow 3 \rightarrow 6$. The fuzzy project duration is the 
Table 3: calculation of total float for each activity in a fuzzy project network and critical path.

\begin{tabular}{|c|c|c|c|c|}
\hline Activity & $1-2$ & $1-3$ & $1-4$ & $2-3$ \\
\hline Duration & $([15,15], 5,5)$ & $([40,40], 10,10)$ & $([20,25], 5,5)$ & $([40,40], 10,10)$ \\
\hline Earliest start & $([0,0], 0,0)$ & $([0,0], 0,0)$ & $([0,0], 0,0)$ & $([15,15], 5,5)$ \\
\hline Earliest finish & $([15,15], 5,5)$ & $([40,40], 10,10)$ & $([20,25], 5,5)$ & $([55,55], 10,10)$ \\
\hline Latest start & $([-50,50], 40,30)$ & $([-35,65], 40,30)$ & $([-20,85], 40,30)$ & $([-35,65], 40,30)$ \\
\hline Latest finish & $([-35,65], 40,30)$ & $([5,1$ top05],40,30) & $([5,105], 40,30)$ & $([5,105], 40,30)$ \\
\hline Total float & $([-50,50], 40,30)$ & $([-35,65], 40,30)$ & $([-20,85], 40,30)$ & $([-50,50], 40,30)$ \\
\hline Activity & $2-5$ & $3-5$ & $4-5$ & \\
\hline Duration & $([100,150], 40,30)$ & $([100,150], 40,30)$ & $([100,150], 40,30)$ & \\
\hline Earliest start & $([15,15], 5,5)$ & $([55,55], 10,10)$ & $([20,25], 5,5)$ & \\
\hline Earliest finish & $([115,165], 40,30)$ & $([155,205], 40,30)$ & $([120,175], 40,30)$ & \\
\hline Latest start & $([5,105], 40,30)$ & $([5,105], 40,30)$ & $([5,105], 40,30)$ & \\
\hline Latest finish & $([155,205], 40,30)$ & $([155,205], 40,30)$ & $([155,205], 40,30)$ & \\
\hline Total float & $([-10,90], 40,30)$ & $([-50,50], 40,30)$ & $([-20,80], 40,30)$ & \\
\hline
\end{tabular}

Table 4: Duration of each fuzzy activity in a fuzzy network problem

\begin{tabular}{|c|c|c|c|c|c|}
\hline Activity & $1-2$ & $1-3$ & $1-5$ & $2-4$ & $2-5$ \\
\hline Duration & $(2,2,3,4)$ & $(2,3,3,6)$ & $(2,3,4,5)$ & $(2,2,4,5)$ & $(2,4,5,8)$ \\
\hline Activity & $3-4$ & $3-6$ & $4-5$ & $4-6$ & $5-6$ \\
\hline Duration & $(1,1,2,2)$ & $(7,8,11,15)$ & $(2,3,3,5)$ & $(3,3,4,6)$ & $(1,1,1,2)$ \\
\hline
\end{tabular}

length of the fuzzy critical path. Hence the optimum fuzzy project duration is $(10,11,14,18)$ fuzzy hours. For this same problem, Usha Madhuri et.al [30] have obtained the same fuzzy critical path 1-3-6 but with the wide fuzzy project duration as $(9,11,14,21)$ fuzzy hours.

\section{Conclusion}

In this paper, the duration hours are considered as imprecise numbers described by trapezoidal fuzzy numbers which are more realistic and general in nature.We proposed a fuzzy version of Critical path method to find the critical path duration for the given problem without converting them to classical one.To illustrate 
Table 5: Duration of each fuzzy activity in a fuzzy network problem

\begin{tabular}{|c|c|c|c|c|c|}
\hline Fuzzy activity & $1-2$ & $1-3$ & $1-5$ & $2-4$ & $2-5$ \\
\hline Duration & $([2,3], 0.1)$ & $([3,3], 1,3)$ & $([3,4], 1,1)$ & $([2,4], 0,1)$ & $([4,5], 2,3)$ \\
\hline Fuzzy activity & $3-4$ & $3-6$ & $4-5$ & $4-6$ & $5-6$ \\
\hline Duration & $([1,2], 0,0)$ & $([8,11], 1,4)$ & $([3,3], 1,2)$ & $([3,4], 0,2)$ & $([1,1], 0,1)$ \\
\hline
\end{tabular}

Table 6: calculation of total float for each activity in a fuzzy project network and critical path.

\begin{tabular}{|c|c|c|c|c|c|}
\hline Activity & $1-2$ & $1-3$ & $1-5$ & $2-4$ & $2-5$ \\
\hline Duration & $(2,2,3,4)$ & $(2,3,3,6)$ & $(2,3,4,5)$ & $(2,2,4,5)$ & $(2,4,5,8)$ \\
\hline Earliest start & $(0,0,0,0)$ & $(0,0,0,0)$ & $(0,0,0,0)$ & $(2,2,3,4)$ & $(2,2,3,4)$ \\
\hline Earliest finish & $(2,2,3,4)$ & $(2,3,3,6)$ & $(2,3,4,5)$ & $(4,4,7,8)$ & $(4,6,8,11)$ \\
\hline Latest start & $(3,4,3,7)$ & $(-1,0,0,4)$ & $(6,7,9,13)$ & $((5,6,6,10)$ & $(4,6,8,12)$ \\
\hline Latest finish & $(5,6,6,10)$ & $(2,3,3,7)$ & $(9,10,13,17)$ & $(7,8,10,14)$ & $(9,10,13,17)$ \\
\hline Total float & $(3,4,3,7)$ & $(-1,0,0,4)$ & $(6,7,9,13)$ & $(3,4,3,7)$ & $(2,4,5,9)$ \\
\hline Activity & $3-4$ & $3-6$ & $4-5$ & $4-6$ & $5-6$ \\
\hline Duration & $(1,1,2,2)$ & $(7,8,11,15)$ & $(2,3,3,5)$ & $(3,3,4,6)$ & $(1,1,1,2)$ \\
\hline Earl. start & $(2,3,3,6)$ & $(2,3,3,6)$ & $(4,4,7,8)$ & $(4,4,7,8)$ & $(6,7,10,12)$ \\
\hline Earl.finish & $(3,4,5,8)$ & $(10,11,14,18)$ & $(6,7,10,12)$ & $(7,7,11,13)$ & $(7,8,11,13)$ \\
\hline Lat.start & $(6,7,8,12)$ & $(2,3,3,7)$ & $(6,7,10,14)$ & $(7,8,10,14)$ & $(9,10,13,17)$ \\
\hline Lat.finish & $(7,8,10,14)$ & $(10,11,14,18)$ & $(9,10,13,17)$ & $(10,11,14,18)$ & $(10,11,14,18)$ \\
\hline Tot.float & $(3,4,5,9)$ & $(-1,0,0,4)$ & $(2,3,3,7)$ & $(3,4,3,7)$ & $(2,3,3,7)$ \\
\hline
\end{tabular}

the proposed method, numerical examples are solved and obtained results are explained. It is to be noted that the result obtained by the proposed method is sharper than the results obtained by the existing methods.

\section{Acknowledgments}

The authors are grateful to the anonymous refrees and the editors for their constructive comments and suggestions. 


\section{References}

[1] Bortolan, G. and Degani, R., A review of some methods for ranking fuzzy subsets,Fuzzy Sets and Systems, 15(1),(1985), 1-19. DOI :10.1016/01650114(85)90012-0

[2] Chanas.S,Kamburowski.J . The use of fuzzy variables in PERT, Fuzzy sets and systems, 5 (1) (1981), 11-19. DOI:10.1016/0165-0114(81)9003-0

[3] Chanas.S,Zielinski.P, Criticalpath Analysis in the networkwith fuzzy activity times, Fuzzy sets and systems, 122(2), (2001), 195-204. DOI:10.1016/S0165-0114(00)00076-2

[4] Chang.S Tsuyimura .Y Tazawa .T, An efficient approach for large scale project planning based on Fuzzy delphi method, Fuzzy sets and systems, 76(3), (1995), 277-288. D0I:10.1016/0165-0114(94)00385-4

[5] Chen, S. H., Ranking fuzzy numbers with maximizing and minimizing set,Fuzzy Sets and Systems, 17(2),(1985), 113-129. DOI:10.1016/01650114(85)90050-8

[6] Chen, Y. L., Rinks, D. and Tang, K., Critical path in an activity network with time constraints, European Journal of Operational Research, 100(1),(1997),122-133. DOI:10.1016/S0377-2217(96)00140-3

[7] Chen, S-P.and Hsue h, Y-J, A simple approach to fuzzy critical path analysis in project networks, Applied Mathematical Modeling, 32(7),(2008), 1289-1297. DOI:10.1016/j.apm.2007.04.009

[8] D.Dubois, H Fargier, V.Galvagonon, On latest starting time and floats in task networks with ill known durations, European journal of Operations Research, 147(2), (2003), 266-280.DOI:10.1016/S0377-2217(02)00560-X

[9] Gazdik.I, Fuzzy network planning FNET, IEEE Transactions reliability, 32(2), (1983), 304-313.DOI:10.1109/TR.1983.5221657

[10] Gonzalez, A., A study of ranking function approach through mean values,Fuzzy Sets and Systems, 35(1), (1990), 29-41. DOI:10.1016/01650114(90)90016-Y

[11] Kaufmann A.Gupta M, Introduction to fuzzy arithmetic theory applications Van Nostrand Reinhold:Newyork, (1985). 
[12] Kim, K. and Park, K. S., Ranking fuzzy numbers with index of optimism, Fuzzy Sets and Systems, 35(2), (1990), 143-150. DOI:10.1016/01650114(90)90189-D

[13] Kuchta D, Use of fuzzy numbers in project risk (criticality) assessment, International journal of project management, 19(5) , (2001), 305-310. DOI:10.1016/S0263-7863(00)00022-3

[14] Levner, E. and Kats, V., A parametric critical path problem and an application for cyclic scheduling, Discrete Applied mathematics, 87(1-3),(1998), 149-158. DOI:10.1016/S0166-218X(98)00054-7

[15] Lin F.T.,Yao J S, Fuzzy Critical path Method based on signed distance ranking of fuzzy numbers, IEEE Transactions on systems $M A N, A N D, C y$ bernetics, 30(1), (2000), 76-82. DOI:10.1109/3468.823483

[16] Lin F.T., Yao J S, Fuzzy critical path method based on signed- distance ranking and statistical confidence -Interval estimates, journal of super computing, 24 (3), (2003), 305-325. DOI:10.1023/A:1022036931014

[17] Liou, T. S. and Wang, M. J. J., Ranking fuzzy number with integral value, Fuzzy Sets and Systems, 50(3),(1992), 247-255. DOI:10.1016/01650114(92)90223-Q

[18] Lorterapong.P Moselhi O, Project network analysis using fuzzy sets theory, Journal of construction engineering and Management, 122(4), (1996), 308-318. DOI:10.1016/(ASCE)0733-9364(1996)122:4(308)

[19] McCahon C.S, Using PERT as an approximations of fuzzy project network analysis IEEE Transaction on Engineering Management, 40 (2), (1993), 146-153. DOI:10.1109/17.277406

[20] Ming Ma, Menahem Friedman and Abraham Kandel. 1999. A new fuzzy arithmetic. Fuzzy Sets and Systems, 108(1),(1999), 83-90. DOI:10.1016/S0165-0114(97)00310-2

[21] Nasution S.H, Fuzzy critical path method, IEEE Transaction on systems MAN,AND Cybernetics, 24(1) (1994), 48-57. DOI:10.1109/21.259685.

[22] N. Ravi Shankar, V. Sireesha and P. Phani Bushan Rao, An Analytical Method for Finding Critical Path in a Fuzzy Project Network, Int. J. Contemp. Math. Sciences, 5 (20),( 2010), 953 - 962. 
[23] Ravi Shankar.N and B. Pardha Saradhi, Fuzzy Critical Path Method in Interval-Valued Activity Networks, Int. J. Pure Appl. Sci. Technol., 3(2), (2011), 72-79.

[24] Shahsavari pour, N., Modarres, M., Aryanejad, M. B., and Tavakoli Moghadam, R., Calculating the project network critical path in uncertainty conditions, International Journal of Engineering and Technology, 2(2), (2010), 136-140.

[25] N. Shahsavari Poura, M. Kheranmandb, M. Fallah c and S. Zeynali d, A new method for critical path method with fuzzy processing time, Management Science Letters, 1(3), (2011), 347-354. DOI:10.5267/j.msi.2011.22.003.

[26] Shakeela Sathish and Ganesan,K., A Simple approach to Fuzzy critical path analysis in project networks, International Journal of Scientific and Research, 2,(2011)

[27] Shih-Pin Chena,A simple approach to fuzzy critical path analysis in project networks, Applied Mathematical Modelling, 32(7), (2008), 12891297. DOI:10.1016/j.apm.2007.04.009

[28] Singh, G. and Zinder, Y., Worst-case performance of critical path type algorithms, InternationalTransactions in Operational Research, 7(4), (2000), 383-399. DOI:10.1111/j.1475-3995.2000.tb00206.X

[29] Slyeptsov, A. I. and Tyshchuk, T. A., A method of computation of characteristics of operations in a problem of fuzzy network, Cybernetics and Planning and Management System Analysis, 39 (3), (2003), 367-378. DOI:10.1023/A:1025705426318

[30] K. Usha Madhuri, B. Pardha Saradhi and N. Ravi Shankar, Fuzzy Linear Programming Model for Critical Path Analysis,Int. J. Contemp. Math. Sciences, 8 (2), (2013), 93 - 116. 
